\title{
DATOS PARA LA PALEONTOLOGÍA CHILENA. LA PALEONTOLOGÍA EN LA EXPEDICIÓN HEULAND A CHILE Y PERÚ (1795-1800)
}

\author{
A. Montero y C. Diéguez \\ Museo Nacional de Ciencias Naturales \\ c/ José Gutiérrez Abascal n. ${ }^{\circ} 2$ - 28006 Madrid (España)
}

\begin{abstract}
RESUMEN
El presente trabajo tiene interés por representar las primeras colectas documentadas de fósiles en territorio chileno, alguna de ellas en zonas hoy bien conocidas de este país. Éstas, se realizaron en la única expedicion totalmente geológica de todas las que llevó a cabo el Estado español a los diferentes virreinatos en los siglos XVIII y XIX. Además de citar el material paleontológico colectado por los expedicionarios, se incluyen los unicos ejemplares paleontológicos de aquella expedicion recuperados por los autores entre los fondos históricos del actual Museo Nacional de Ciencias Naturales de Madrid, España.
\end{abstract}

\section{SUMMARY}

This paper deals with the first paleontological collecting in Chile in sites well known today. These collectings were carried out in the sole totally geological expedition of those undertook by the Spanish state during the XVIII and XIX centuries. All the paleontological material collected by the expedition members is listed, incluiding the specimens retrieval by the authors amongst the historical collections of the present Museo Nacional de Ciencias Naturales of Madrid, Spain.

\section{INTRODUCCIÓN}

La Expedicion Heuland (1795-1800) ha sido ya clasificada como expedición mineralógica (Guirao, 1988), y también como una expedición de iniciativa particular y de carácter nacional (Del Pino y Guirao, 1988). La ruta seguida por los expedicionarios, la formación científica que poseían y los resultados de sus colectas no ofrecen dudas al respecto en cuanto a su clasificación como expedición geológica o mineralógica (Gutiérrez, 1987), si bien los fines perseguidos inicialmente comprendían también otras ramas de la Historia Natural (el apartado $2^{\circ}$ de la Instrucción Real de colecta obligaba a los expedicionarios a recoger, entre otras cosas, "petrificaciones"). 
Estuvo formada inicialmente por los hermanos Cristian y Conrad Heuland, que se encargarían de la parte geológica y malacológica de la expedición, y por Francisco Javier Molina que se ocuparía de la parte zoológica excepto la malacológica ${ }^{1,2,3}$. Este último, no llegó a tomar parte en el viaje por lo que la parte a él encomendada quedaba sin cubrir, y en cuanto a la malacología, el itinerario seguido por los hermanos Heuland no era muy propicio para la recolección de estos ejemplares (rutas mineras), aunque visitaron la costa argentina y chilena en varias ocasiones (Barreiro, 1929a; 1929b; 1930; Arias Divito, 1978).

Por tanto, la expedición acabó realizando un recorrido mineralógico, fundamentalmente por el territorio del actual Chile durante los dos primeros años, y Bolivia y Perú a partir de 1798, aunque esta última parte del viaje es mucho menos conocida.

\section{RESULTADOS PALEONTOLÓGICOS DE LA EXPEDICIÓN HEULAND}

En la documentacion manejada por nosotros, aparecen varios documentos en los que Cristian Heuland avisa al Real Gabinete de Madrid del envío de fósiles colectados o comprados en su recorrido (Fig. 1). Los primeros fósiles los colectaron o más probablemente compraron durante la primera parte del viaje, entre febrero y marzo de 1795, en su escala de varios meses en Buenos Aires y al menos uno de ellos es de la Patagonia. En un primer documento, C. Heuland dice mandar:

\footnotetext{
"Pétrification d'un Crustacé, c'est un Crabe amphibie de conservation assez parfaite, dont les interstices de ses articulations sont remptis de matière terrestre sabloneuse-grenue. 6 pouces sur 4 du Port $S^{t} J o s e p h$ a la Côté Patagone" y también: "Osement fossile spongieux et poreux d'un animal amphibie,. ainsi disposé et preparé par la nature pour recevoir le suc lapidifique ou les molecules terrestres pour se pétrifier. Cette pièce qui a son mérite, paroitêtre une portion organique de la tête et du col d'un petit crocodile connu en cette Amérique sous les noms de Cayman et de Jacara"4.
}

1 13-Abr.-1793. Documento número 660 del Catálogo de las expediciones y viajes científicos españoles. Siglos XVIII y XIX. CALATAYUD, A. (1984). Museo Nacional de Ciencias Naturales-CSIC, 433 pp. Madrid.

2 12-Jun.-1794. Documento número 664 del Catálogo de las expediciones y viajes científicos españoles. Siglos XVIII y XIX. CALATAYUD, A. (1984). Museo Nacional de Ciencias Naturales-CSIC, 433 pp. Madrid.

3 17-Sep.-1794. Documento número 664 del Catálogo de las expediciones y viajes científicos españoles. Siglos XVIII y XIX. CALATAYUD, A. (1984). Museo Nacional de Ciencias Naturales-CSIC, 433 pp. Madrid.

4 18-Mar.-1795. Documento número 667 del Catálogo de las expediciones y viajes científicos españoles. Siglos XVIII y XIX. CALATAYUD, A. (1984). Museo Nacional de Ciencias Naturales-CSIC, 433 pp. Madrid. 


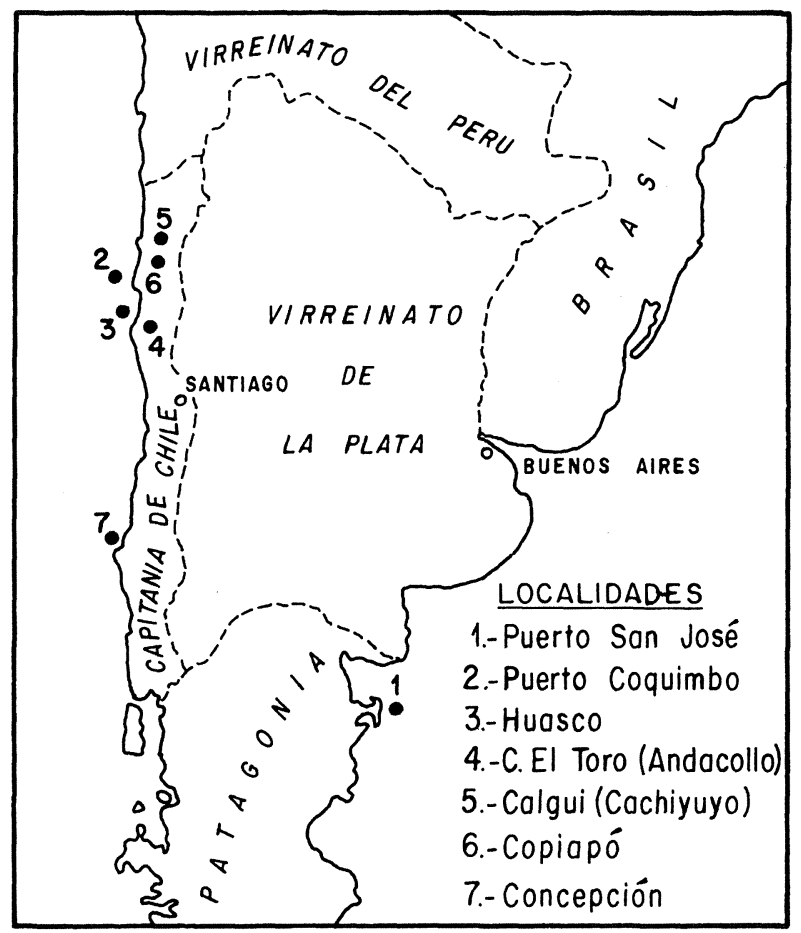

Fig. 1. Localidades conocidas de colecta o adquisiciones de ejemplares fósiles en la Expedición Heuland.

Las siguientes referencias de colectas de petrificaciones en la documentacion de esta expedición se encuentran en los listados de los ejemplares enviados a la corte española y son ya todas de territorio chileno:

"Coquilles pétrifiées du Guasco: Deux Peignes pétrifiés et spathifiés, à côtes saillantes; imparfaites du côté inférieur. Le $\mathrm{N}^{\circ} 2$ est plus comprimé; Huit Cames feuilletées, à conches superposées; petrifiées et spathifiées."

"Petrifications du Port de Coquimbo: Quatre Pieces qui sont autant de jointures de l'epine du dos d'une Baleine, pétrifiée et a l'etat de pierre calcaire. Elles sont très bien articulées et leur conservation ne laisse rien desirer. Nous les retirames d'une fouille que nous fimes faire prés du rivage au Port de Coquimbo. On peut les considérer comme fort-rares." 5

5 12-Feb.-1797. Documento número 670 del Catálogo de las expediciones y viajes científicos españoles. Siglos XVIII y XIX. CALATAYUD, A. (1984). Museo Nacional de Ciencias Naturales-CSIC, 433 pp. Madrid. 


\begin{abstract}
"Province de Copiapó: Grand Limaçon pétrifié, pyramidal à contours saillants et cannelé dans son circonferance: avec terre jaune calcaire. 4 pouces de longueur sur $2 \&$ demi de large vers sa bouche de la Cordillére au dessus d'un Lieu de mines nommé Calgui: à 38 lieus NE. de Copiapó; Limaçon petrifié de la même espèce, moins gros. Plus de 3 pouces sur 2. La même Limaçon à rainures multipliées et fort saillantes, argué par intervalles et fortement applation comprimé. 3 pouces et demi sur $2 \&$ demi. Grand Peigne pétrifié dont la valve supérieure a grosses et larges côtes saillantes, convexes, et l'infeérieure à profondes cannelures de la Montagne del Toro." 6
\end{abstract}

Por último, en documentos de 1806 se citan varias petrificaciones recogidas en esta expedición: das."

"Copiapó: Diez y seis papelitos con petrificaz. de conchas $\&^{\mathrm{a}}$. Dos conchas petrifica-

Y tambien:

Un pedazo de madera petrificada; dos conchas grandes $\mathrm{Y}$ veinte y dos pequeñas, todas petrificadas: cinquenta y cinco conchas medianas petrificadas y sesenta y dos idem mui pequeñas." 8,9

En estas últimas referencias no se aporta localidad de origen.

Como se puede observar por las localidades de colecta, el viaje chileno fue el que reportó los únicos fósiles a excepción del que consiguieron de la costa patagona, dentro de lo que hoy es Argentina. Es curioso que los expedicionarios buscaran yacimientos de fósiles en zonas mineras, con grandes afloramientos de rocas plutónicas y volcánicas tanto jurásicos como cretácicos y terciarios, aunque asociadas a niveles sedimentarios, y donde lógicamente la presencia de yacimientos fosilíferos queda marginada por el interés metalogenético de las áreas visitadas (Oyarzun et al, 1987).

6 12-Feb.-1797. Documento número 670 del Catálogo de las expediciones y viajes científicos españoles. Siglos XVIII y XIX. CALATAYUD, A. (1984). Museo Nacional de Ciencias Naturales-CSIC, 433 pp. Madrid.

7 Oct.-1806. Documento número 678 del Catálogo de las expediciones y viajes científicos españoles. Siglos XVIII y XIX. CALATAYUD, A. (1984). Museo Nacional de Ciencias Naturales-CSIC, 433 pp. Madrid.

8 18-Oct.-1806. Documento número 678 del Catálogo de las expediciones y viajes científicos españoles. Siglos XVIII y XIX. CALATAYUD, A. (1984). Museo Nacional de Ciencias Naturales-CSIC, 433 pp. Madrid.

9 21-Oct.-1806. Documento número 678 del Catálogo de las expediciones y viajes científicos españoles. Siglos XVIII y XIX. CALATAYUD, A. (1984). Museo Nacional de Ciencias Naturales-CSIC, 433 pp. Madrid. 
Sirva como ejemplo, los bivalvos recogidos en el Cerro del Toro (Neocomiense, Formación Arqueros o Formación Quebrada Marqueza Miembro I), cerca de Andacollo en donde el interés fundamental reside en las cercanas vetas auríferas y cupríferas (Llaumet et al, 1975).

Algunas localidades mencionadas en la documentación de esta expedicion han sido posteriormente redescubiertas, convirtiéndose en yacimientos bien conocidos en la Paleontología chilena. Así, las "cuatro piezas de la espina de ballena" que los Heuland afirman colectar en el Puerto de Coquimbo es el primer antecedente de descubrimientos posteriores de cetáceos (Salinas, 1988). También es posible que los moluscos marinos que tienen como localidad Huasco o Copiapó, sin mas especificaciones, puedan pertenecer a algún yacimiento conocido actualmente entre estas dos poblaciones o cerca de alguna de ellas (Tavera, 1956; Covacevich, 1985; Cooper et al, 1989; Pérez y Reyes, 1989; Pérez et al, 1990).

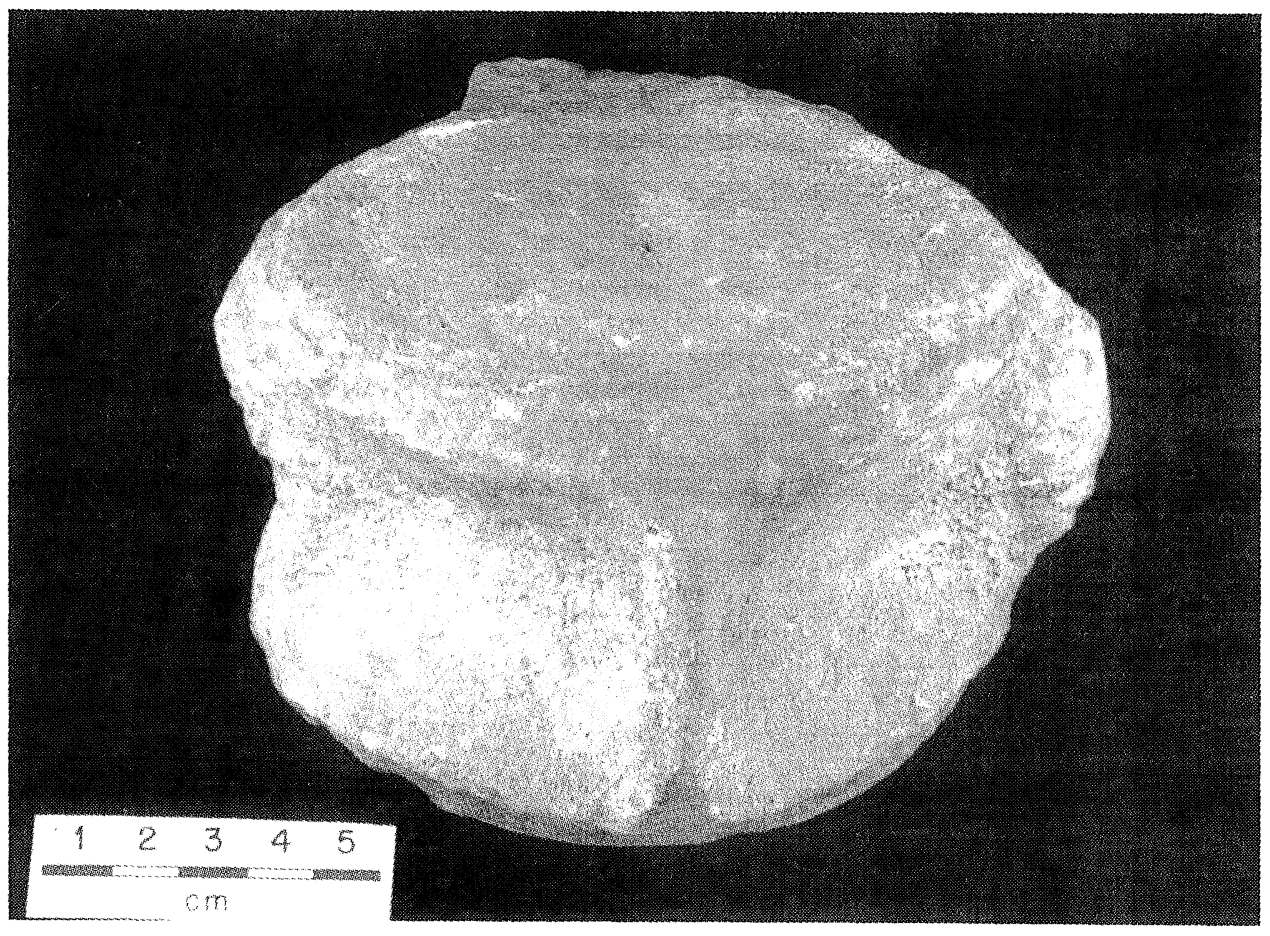

Fig. 2. Vértebra de cetáceo del Puerto de Coquimbo, Chile. 


\section{EJEMPLARES RECUPERADOS DE LA EXPEDICIÓN HEULAND}

De todo el material colectado o adquirido por los hermanos Heuland en este viaje, en la actualidad se han recuperado de los fondos históricos de lo que fue el Real Gabinete de Historia Natural, actual Museo Nacional de Ciencias Naturales, un crustáceo (Decapoda, Zanthopsidae) que se encontraba determinado como Cancrites sp., tratándose realmente de un Harpactoxanthopsis sp. (MNCNI-18009), del Eoceno (Collins, 1991, com. escrita), y que corresponde al ejemplar reseñado en la primera carta de los hermanos Heuland como "petrificacion de un crustáceo..." (Fig. 2). Asimismo, cuatro vértebras (MNCN-9355) de la colección histórica presentan la etiqueta

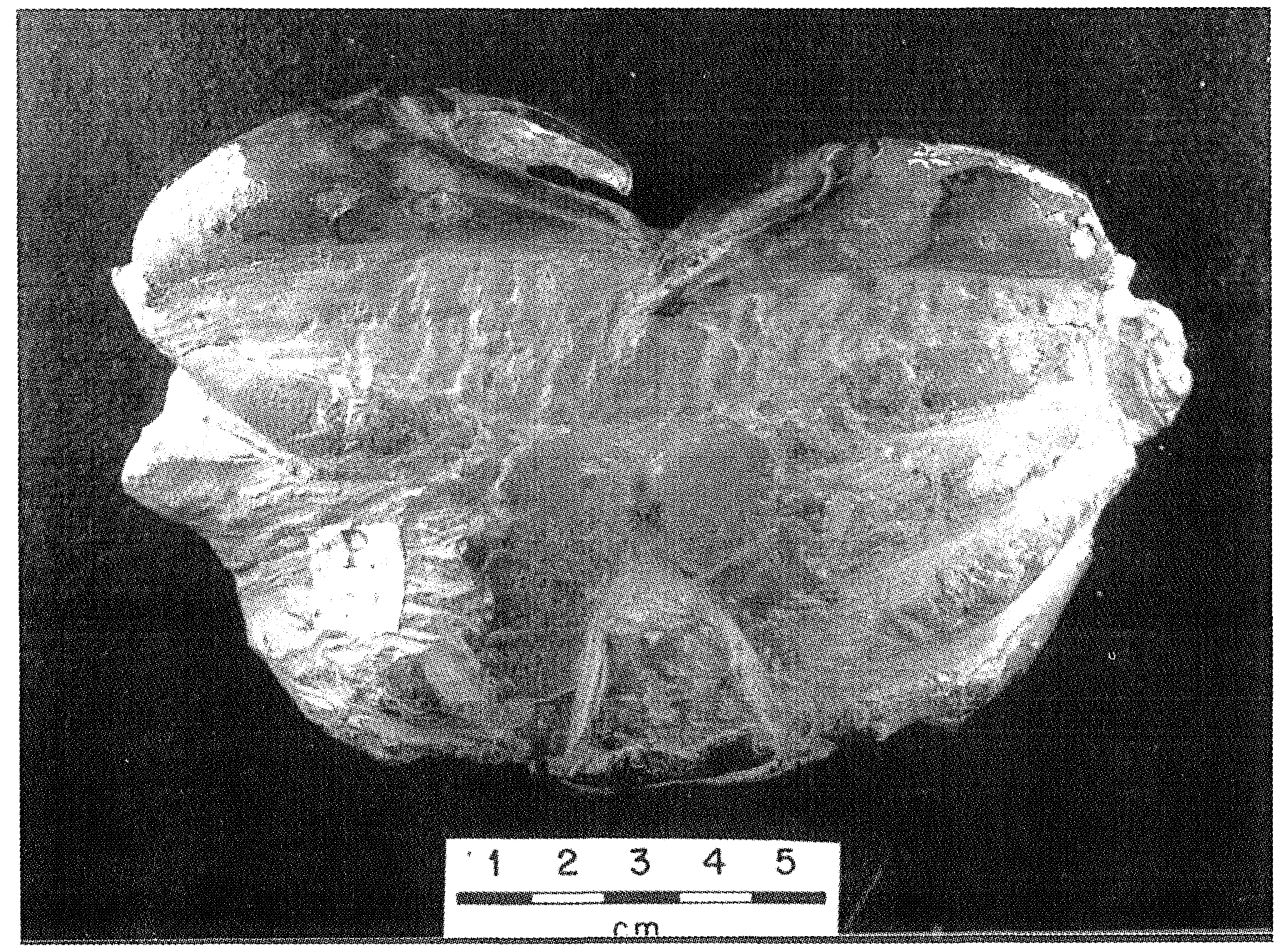

Fig. 3. Harpactoxanthopsis sp. del Eoceno del Puerto de San José, Costa Patagona, Argentina.

característica de esta expedición, con la sigla correspondiente a las vértebras de ballena del Puerto de Coquimbo colectadas por los expedicionarios (Fig. 3) y, aunque dentro 
de la colección habían sido reclasificadas como vértebras de dinosaurio ha sido confirmada su pertenencia a los cetáceos (J. L.Sanz, 1996, com. pers.). Un tronco fósil de la colección histórica (MNCNV-1034) presenta una etiqueta de esta expedicion y puede corresponder al ejemplar de "madera petrificada..." sin localidad específica (Fig. 4). Otro tronco fósil (MNCNV-1025) que se encuentra también entre las colecciones antiguas de este Museo tiene como localidad de origen la Plaza del Nacimiento

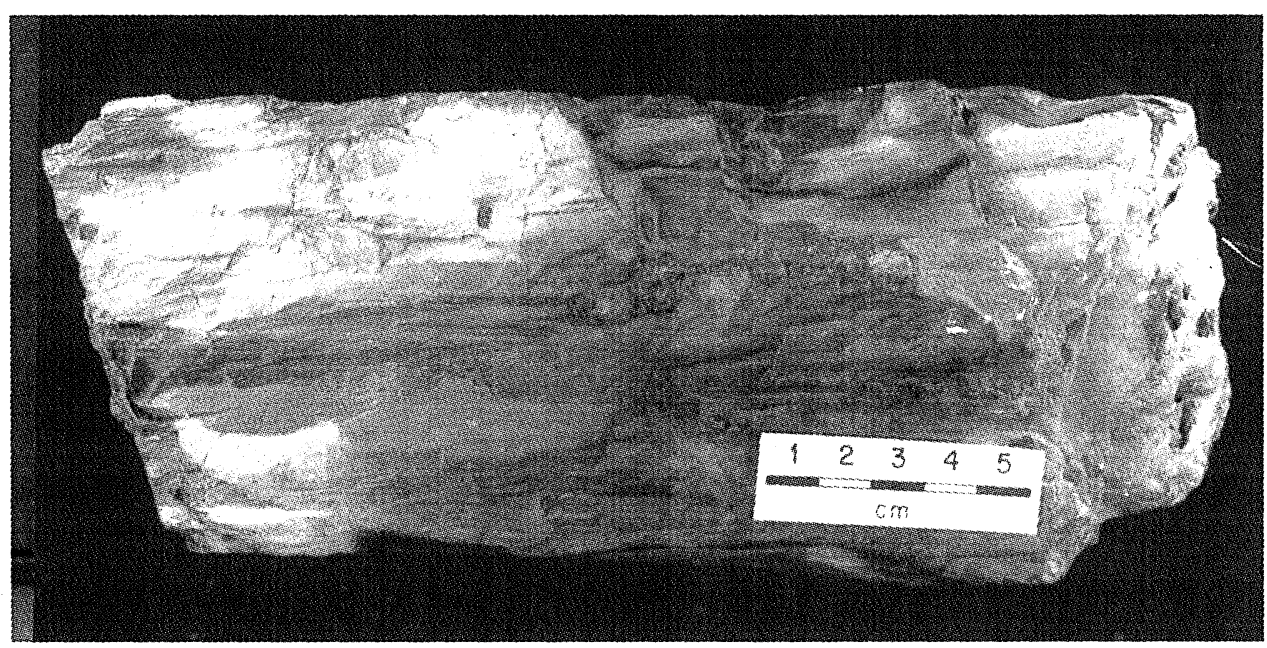

Fig. 4. Leño fósil sin localidad específica. Chile.

(Obispado de la Concepción, Chile) y podría pertenecer a una compra de esta expedición (Fig. 5), aunque no conserva etiquetas originales. Y lo mismo ocurre con un ejemplar incompleto de bivalvo (Trigoniidae) datado históricamente como cretácico (MNCNI-27852) y dando Chile como único dato de localidad (Fig. 6). En estos dos últimos casos, no descartamos que pudieran pertenecer a esta expedición dado la escasez de material de Chile en el Museo Nacional de Ciencias Naturales de Madrid y la prácticamente inexistencia de colectas en ese país, salvo en lo que se refiere a colectas de expediciones históricas (Montero, 1995). 
A. MONTERO Y C. DIÉGUEZ

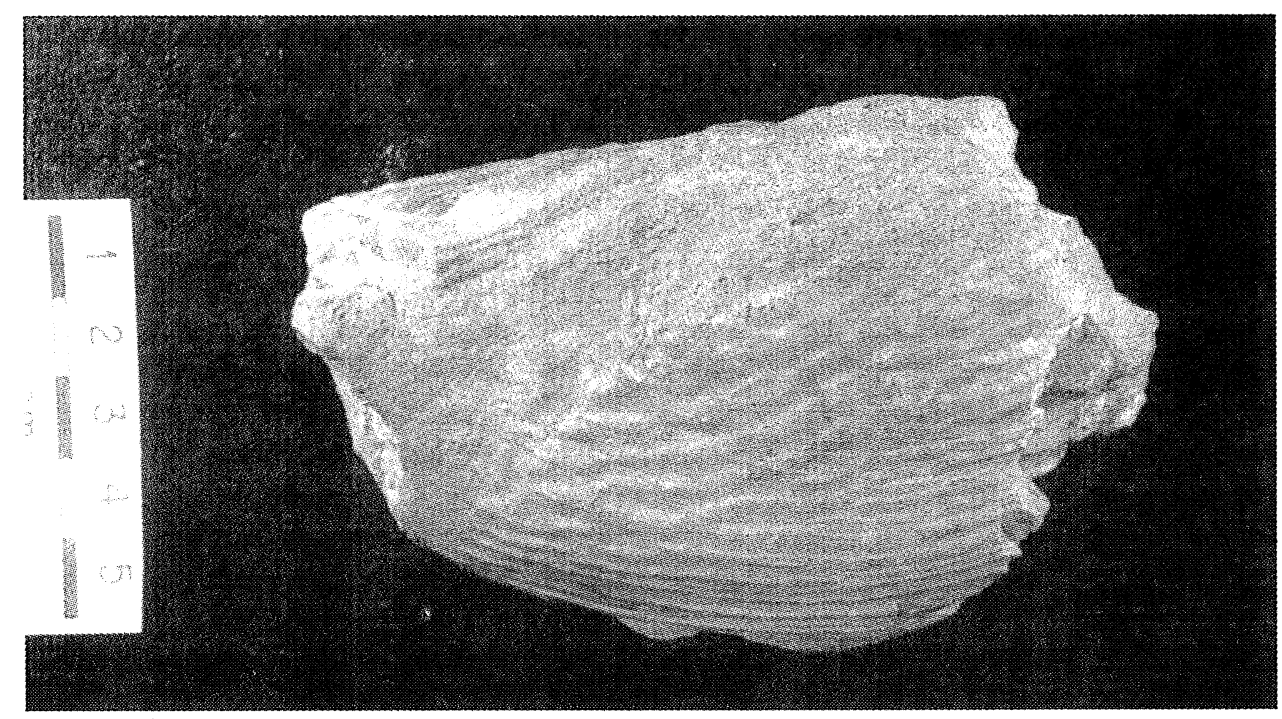

Fig. 5. Ejemplar de Bivalvo Trigoniidae sin localidad específica. Chile.

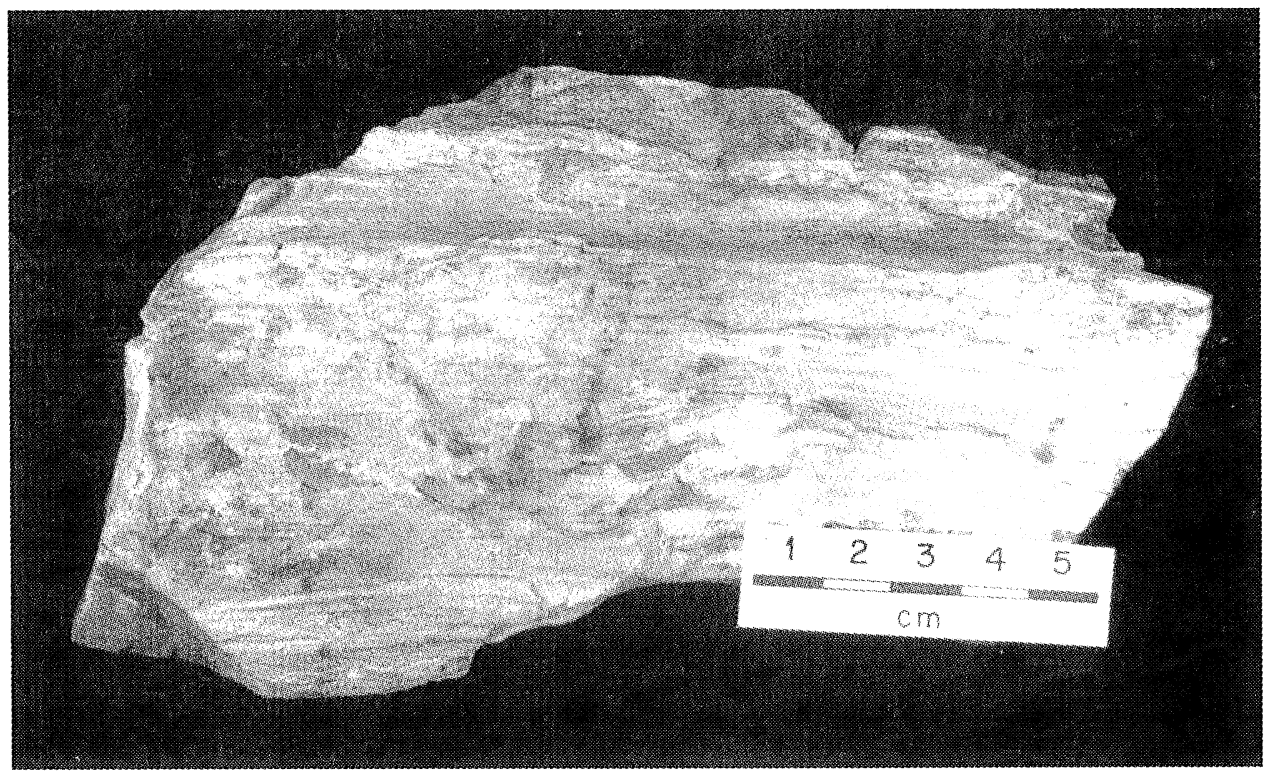

Fig. 6. Leño fósil de la Plaza del Nacimiento, Obispado de la Concepción, Chile. 


\section{AGRADECIMIENTOS}

Los autores quieren agradecer al Dr. Javier García Guinea las ideas aportadas al manuscrito que lo enriquecieron considerablemente. También nuestro agradecimiento al departamento de fotografía y al personal de delineación del Museo Nacional de Ciencias Naturales de Madrid.

\section{CONCLUSIONES}

La expedición que realizaron los hermanos Heuland a finales del siglo XVIII fue la única expedición totalmente geológica que llevó a cabo la corona española. La finalidad primordial de esta expedición fue la mineralógica, centrada en las menas metálicas, fundamentalmente chilenas, bolivianas y peruanas, aunque también colectaron y adquirieron fósiles, ya que ésta era una finalidad de la expedición, aunque quedaba supeditada al recorrido mineralógico.

Los descubrimientos fosilíferos en territorio chileno que aparecen en la documentación de esta expedición constituyen las primeras referencias de yacimientos paleontológicos en ese país, algunos de los cuales son actualmente bien conocidos, como queda reflejado en la literatura paleontológica chilena.

Hay que señalar que si bien los primeros años del viaje han sido muy conocidos por existir documentación de la época, los tres últimos años son prácticamente desconocidos por la ausencia de ésta. Así, es lógico que Calderón (1892) escribiera que aunque los hermanos Heuland debieron recoger numerosos ejemplares, se desconocía la existencia de catálogos, fechas de envíos, etc., lo que lógicamente dificultaba su localización. A pesar de esto, se han recuperado entre los fondos históricos del Museo Nacional de Ciencias Naturales de Madrid algunos ejemplares de esta expedición.

\section{BIBLIOGRAFÍA}

ARIAS Divito, J. C. (1978). Expedición científica de los hermanos Heuland (1795-1800). Ed. Cultura Hispánica, 150 p., Madrid.

Barreiro, A. (1929 a). "El viaje científico de Conrado y Cristiano Heuland a Chile y Perú, organizado por el Gobierno español en 1795" (1. " parte). Bol. R. Soc. Geográfica de Madrid, vol. 69, pp. 157-199.

Barreiro, A. (1929 b). "El viaje científico de Conrado y Cristiano Heuland a Chile y Perú, organizado por el Gobierno español en 1795" (2. a parte). Bol. R. Soc. Geográfica de Madrid, vol. 69, pp. 425-466 


\section{A. MONTERO Y C. DIÉGUEZ}

Barreiro, A. (1930). "El viaje científico de Conrado y Cristiano Heuland a Chile y Perú, organizado por el Gobierno español en 1795" (3. a parte). Bol. R. Soc. Geográfica de Madrid, vol. 70, pp. 269-294.

CALderón, S. (1892). Los naturalistas españoles en América. Ed. F. Santiago, 63 pp. Sevilla.

COOPER, M. R.; PÉREZ, E., y REYES, R. (1989). "Systematic position of Trigonia nepos Paulcke, 1903 and Paulakella, a new genus for the Aptian of Chile", Rev. Geológica de Chile, vol. 16, n. ${ }^{\circ}$, pp. 51-59.

Covacevich, V. (1985). "Nueva localidad para Myophorella (M) hillebrandti Reyes y Pérez en el norte de Chile: significado cronológico y paleobiogeográfico". Rev. Geológica de Chile, n. ${ }^{\circ} 24$, pp. 103-106.

GutiéRrez, E. (1987): "Preparativos de la expedición de los hermanos Heuland a Chile y Perú”. En: La expedición mineralógica de los hermanos Heuland a Chile y Perú. 1795-1800. Ed. Comisión V Centenario, Museo Nacional de Ciencias Naturales-CSIC, pp. 29-38, Madrid.

GuIRAO, A. (1988). "Notas para la clasificación de las expediciones españolas del siglo XVIII a América". En: IV Congreso Soc. Esp. de Historia de las Ciencias y de las Técnicas, 1986, vol. II, pp. 585-595, Valladolid.

Llaumet, C.; OlCay, L.; Marquardt, J. C., y Reyes, E. (1975). "El yacimiento de cobre porfídico 'Andacollo', provincia de Coquimbo, Chile”, Rev. Geológica de Chile, n. 2, pp. 56-66.

MONTERO, A. (1995). La colección de invertebrados fósiles del Museo Nacional de Ciencias Naturales de Madrid-CSIC. Su desarrollo histórico y museológico. Tesis doctoral (inédita), Universidad Complutense de Madrid, Facultad de Ciencias Geológicas, Departamento de Paleontología, $807 \mathrm{pp}$.

OyarzúN, R., Martínez FríAs, J., y García GuinEA, J. (1987). "Expedición Heuland (17951800). La minería de la época dentro del contexto de la metalogenia actual". En: La expedición mineralógica de los hermanos Heuland a Chile y Perú. 1795-1800, Ed. Comisión V Centenario, Museo Nacional de Ciencias Naturales-CSIC, pp. 53-80, Madrid.

PÉREZ, E, y REYES, R. (1989). "Dos nuevas especies del género Paulckella Cooper et al, 1989 (Bivalvia, Trigoniidae), de Cretácico Inferior, Norte de Chile, Rev. Geoglógica de Chile, vol. 16, n..$^{\circ}$, pp. 217-227.

PÉrez, E.; CoOPer, M. R., y Covacevich, C. (1990). “Aptian Ammonite-based age for the Pabellon Formation, Atacama Region, Northern Chile", Rev. Geológica de Chile, vol. 17, n. . 2, pp. $181,185$.

Pino DíAZ, F. DEL, y GuiraO, A. (1988). "Las expediciones ilustradas y el estado español”. En: Ciencia y contexto histórico nacional en las expediciones ilustradas a América (Coord. F. DEL PINO), Dep. Historia de América, Centro de Estudios Históricos-CSIC, pp. 19-69, Madrid.

Salinas, P. (1988). “Hallazgo de cetáceos fósiles (MYsticeti, Balaenopteridae) en la ciudad de Coquimbo, Chile", Rev. Geológica de Chile, vol. 15, n. ${ }^{\circ}$ 1, pp. 89-94.

Tavera, J. (1956). "Fauna del Cretáceo Inferior de Copiapó", Anales de la Facultad de Ciencias Físicas y Matemáticas, vol. 13, pp. 205-216. 